\title{
Reparametrization invariance: a gauge-like symmetry of ultrametrically organised states
}

\author{
T. Temesvári \\ Institute for Theoretical Physics, Eötvös University, \\ H-1117 Pázmány Péter sétány 1, Bld. A, Budapest, Hungary \\ I. Kondor \\ Department of Physics of Complex Systems, Eötvös University, \\ H-1117 Pázmány Péter sétány 1, Bld. A, Budapest, Hungary \\ C. De Dominicis \\ Service de Physique Théorique, CEA Saclay, \\ F-91191 Gif sur Yvette, France
}

November 6, 2018

\begin{abstract}
The reparametrization transformation between ultrametrically organised states of replicated disordered systems is explicitly defined. The invariance of the longitudinal free energy under this transformation, i.e. reparametrization invariance, is shown to be a direct consequence of the higher level symmetry of replica equivalence. The double limit of infinite step replica symmetry breaking and $n \rightarrow 0$ is needed to derive this continuous gauge-like symmetry from the discrete permutation invariance of the $n$ replicas. Goldstone's theorem and Ward identities can be deduced from the disappearence of the second (and higher order) variation of the longitudinal free energy. We recall also how these and other exact statements follow from permutation symmetry after introducing the concept of "infinitesimal" permutations.
\end{abstract}

\section{Introduction}

Exact statements such as Goldstone's theorem [1, 2] and Ward identities [3] proved very useful in the perturbative analysis of ordinary statistical systems with a continuous symmetry. For example, Goldstone's theorem ensures that the low temperature phase of the $\mathrm{O}(\mathrm{m})$ model is massless 
(the transverse susceptibility is infinite), while the Ward identities, i.e. the exact relations between the different vertex functions (derivatives of the Legendre-transformed free energy), help us to get rid of dangerous infrared divergences, order by order in perturbation theory [4].

Zero modes and massless phases are surprisingly frequent in quenched random systems, too. The idea of the spin glass phase being marginal occured very early to various authors [5, 6] studying the mean field version of the Ising spin glass, the Sherrington-Kirkpatrick (SK) model [7], without using replicas. After the solution of the SK model by Parisi [8], the marginality of one of the most dangerous eigenvalues across the whole spin glass phase was proven by two different technics [9, 10]. In the truncated version of the SK model, valid close to $T_{c}$, a whole band of zero and near-zero eigenvalues were found [11. The nature of the spin glass phase in finite dimensional models with short range interaction has been the subject of intensive debate, and the question has not been settled until now. Nevertheless, both conflicting theories, "droplet" picture or phenomenological scaling on one side [12, 13, 14] and the ultrametrically organised complex phase space structure of Parisi on the other [8, 15, 16], predict a marginal spin glass phase with an infinite spin glass susceptibility.

To give another example of a quenched disordered system with a massless low temperature phase, we can mention the long-range correlated random manifold problem in $d=D+N$ dimensions, where $D$ is the intrinsic dimension of the manifold. For $N \rightarrow \infty$, while $D<4$ is kept fixed, the zero momentum limit of a family of eigenvalues of the mass operator goes to zero [17]. (In the notation of Ref. [17], $\lambda_{\vec{p}=0}(x ; x, x)=0,0 \leq x \leq 1$, where the momentum vector $\vec{p}$ is $D$ dimensional.)

The idea that a continuous symmetry emerges in the Ising spin glass, which is a discrete model, giving rise to Goldstone modes was raised twenty years ago 18. Bray and Moore argued, that it is the replica limit $n \rightarrow 0$, which may be responsible for this rather strange phenomenon. In Sec. 2, we will recall how the permutation invariance of the $n$ replicas becomes a continuous symmetry in systems whose ordered phase is organised in the hierarchical way proposed by Parisi, but only in the limit $R \rightarrow \infty$, where $R$ is the number of steps in Parisi's construction. All the examples above belong to this class, except the "droplet" theory, which corresponds to a replica symmetric $(R=0)$ picture. The symmetry argument we will use applies for a replicated system, and is a direct consequence of the replica trick. How to derive Goldstone's theorem in the original models and what is the continuous symmetry there, is not clear for us and remains an open question. The results of Sec. 2 were derived earlier using different, though closely related, infinitesimal permutations [19].

The main result of this paper is left to Sec. 3, where it is shown how the somewhat misterious reparametrization invariance 15] follows from the permutation symmetry of the $n$ replicas. Ward identities can then be derived, at least in principle, from the disappearance of the second, third, etc. variations of the free energy funcional. We have thus two different methods to obtain Ward identities: invariance under infinitesimal permutations and/or reparametrization. To make the presentation clear, computational details are left to the Appendix. 


\section{Permutation symmetry and Ward identities}

Our starting point is the free energy $F$, expressed as a functional of the order parameters $q_{\alpha \beta}$, $\alpha, \beta=1,2, \ldots, n$ and $\alpha<\beta$, with $n$ the replica number. The order parameters $q_{\alpha \beta}$ are usually regarded as the elements of a real, symmetric matrix, zero along the diagonal $\left(q_{\alpha \beta}=q_{\beta \alpha}\right.$, $\left.q_{\alpha \alpha}=0\right)$; for our purposes it will be useful to think of them as components of a $\frac{1}{2} n(n-1)$ dimensional vector $\vec{q}$. The association between the matrix $q_{\alpha \beta}$ and the vector $\vec{q}$ is obvious: one lists the elements of $q_{\alpha \beta}$ with $\alpha<\beta$ in any prescribed order (say row by row) to form a column vector.

Although, the symmetry arguments we are going to use are completely general, we wish to give here some specific examples. The reader may think of $F(\vec{q})$ as

- the functional appearing in the integral representation of the quenched averaged free energy of the SK model [16];

- the Legendre-transformed free energy, with respect to a source $h_{\alpha \beta}$, of a replica field theory with a Lagrangean $\mathcal{L}\left(\phi_{\alpha \beta}\right)$ invariant under any permutation of the $n$ replicas;

- a functional obtained by a second Legendre-transformation [20] of a replica field theory with a Lagrangean $\mathcal{L}\left(\phi_{\alpha}\right)$ (the source $h_{\alpha \beta}$ now couples to products like $\left.\phi_{\alpha} \phi_{\beta}\right)$. In this case the notation $G_{\alpha \beta}$ is preferred to $q_{\alpha \beta}$. The random field Ising model [21] and the random manifold problem [17, 22] are good examples where such a functional has an important role. Although the diagonal elements $G_{\alpha \alpha}$ are no longer zero now, this leads to only slight modifications in the arguments and has no influence on the results.

By construction, the free energy is invariant with respect to the permutations $P$ of the replicas:

$$
F\left(\vec{q}^{\prime}\right)=F(\vec{q}) \quad \text { for } \quad q_{\alpha \beta}^{\prime}=q_{P_{\alpha} P_{\beta}} .
$$

Permuting the components of a vector will not change its length, so $P$ generates an orthogonal transformation:

$$
\vec{q}^{\prime}=\mathbf{O} \vec{q}, \quad \mathbf{O}^{\mathrm{T}}=\mathbf{O}^{-1} .
$$

The invariance of $F$ under $\mathbf{O}$ implies that its gradient transforms as a vector:

$$
\frac{\partial F}{\partial \vec{q}^{\prime}}=\mathbf{O} \frac{\partial F}{\partial \vec{q}}
$$

Throughout this paper, Eq. 11 will be used for a vector $q_{\alpha \beta}$ built up by Parisi's hierarchical construction [8, 16], and having the following properties: The (for the time being) discrete values of the matrix elements will be called $q_{r}$, and the sizes of the hierarchically arranged blocks $p_{r}$, $r=0,1, \ldots, R+1$ and, by convention, $p_{0}=n$ and $p_{R+1}=1 . r$ is the overlap of the replicas $\alpha$ and 
$\beta, r=\alpha \cap \beta$, i.e. $q_{\alpha \beta}=q_{r}$, and by definition $\alpha \cap \alpha=R+1$. Both series of parameters are assumed to be monotonic: $q_{s}<q_{r}$ and $p_{s}<p_{r}$ for $s<r$. $R$ is the number of replica symmetry breaking steps; our main concern will be the evolution of the symmetries of the system as $R \rightarrow \infty$. For large but finite $R$ the $q$ 's and the $p$ 's fill the intervals $\left[q_{0}, q_{R}\right]$ with $q_{0} \geq 0, q_{R}<1$, and $\left[p_{1}, p_{R}\right]$ with $p_{1}>p_{0}=n \geq 0, p_{R}<1$, respectively, in a quasi continuous manner:

$$
\begin{aligned}
q_{r+1}-q_{r} & =O(1 / R), & r & =0,1, \ldots, R-1, \\
q_{r+1}+q_{r-1}-2 q_{r} & =O\left(1 / R^{2}\right), & & r=1,2, \ldots, R-1 \\
p_{r+1}-p_{r} & =O(1 / R), & & r=1,2, \ldots, R-1 \\
p_{r+1}+p_{r-1}-2 p_{r} & =O\left(1 / R^{2}\right), & & r=2,3, \ldots, R-1
\end{aligned},
$$

(the difference $p_{1}-p_{0}$ and $p_{R+1}-p_{R}$ may be of $O(1)$ ). A vector $\vec{q}$ associated with a matrix $q_{\alpha \beta}$ with the above structure and continuity properties as in Eq. 2 will, in the following, be called a Parisi vector. Note that we have not assumed the $q$ 's and $p$ 's to be stationary. In fact, in most of what follows we will be considering symmetries that are present irrespective of whether we are at a stationary point or not.

Let us consider now the action of a special permutation of replicas on a Parisi vector. The permutation will be chosen in such a way as to interchange two blocks of size $p_{r+2}$ and leave the rest unchanged. The replicas belonging to these two blocks will be labelled by $\alpha_{i}$ and $\beta_{i}$, respectively, $i=1,2, \ldots, p_{r+2}$. The permutation in question will then act as $P_{\alpha_{i}}=\beta_{i}$ and $P_{\beta_{i}}=\alpha_{i}$ for $i=1,2, \ldots, p_{r+2}$, and as $P_{\alpha}=\alpha$ for $\alpha$ outside the two selected blocks. If the two blocks belong to the same block of size $p_{r+1}$, i.e. if $\alpha_{i} \cap \beta_{i}=r+1$, this permutation is just an element of the residual symmetry group that remains after replica symmetry breaking (RSB), and will leave the Parisi vector invariant $\left(\vec{q}^{\prime}=\vec{q}\right)$. If the two blocks are chosen farther apart $\left(\alpha_{i} \cap \beta_{i}<r+1\right)$, however, $\vec{q}^{\prime}$ will not be a Parisi vector any more, and, depending on $P$, a smaller or larger difference $\vec{q}^{\prime}-\vec{q}$ develops. The smallest change is expected for $\alpha_{i} \cap \beta_{i}=r$. For this choice, the only nonzero components of $\vec{q}^{\prime}-\vec{q}$ are the following:

$$
\left(\vec{q}^{\prime}-\vec{q}\right)_{\alpha_{i} \gamma}=\left(\vec{q}^{\prime}-\vec{q}\right)_{\beta_{i} \delta}=q_{r+1}-q_{r},
$$

for

$$
\alpha_{i} \cap \gamma=\beta_{i} \cap \delta=r \quad \text { and } \quad \alpha_{i} \cap \delta=\beta_{i} \cap \gamma=r+1 \quad \text {, }
$$

and

$$
\left(\vec{q}^{\prime}-\vec{q}\right)_{\alpha_{i} \gamma}=\left(\vec{q}^{\prime}-\vec{q}\right)_{\beta_{i} \delta}=q_{r}-q_{r+1},
$$

for

$$
\alpha_{i} \cap \gamma=\beta_{i} \cap \delta=r+1 \quad \text { and } \quad \alpha_{i} \cap \delta=\beta_{i} \cap \gamma=r \quad .
$$

The length of $\vec{q}^{\prime}-\vec{q}$ is

$$
\sqrt{\left(\vec{q}^{\prime}-\vec{q}\right)^{2}}=\sqrt{\sum_{\alpha<\beta}\left(\vec{q}^{\prime}-\vec{q}\right)_{\alpha \beta}^{2}}=\sqrt{4 p_{r+2}\left(p_{r+1}-p_{r+2}\right)\left(q_{r+1}-q_{r}\right)^{2}}
$$


which is of $O\left(1 / R^{3 / 2}\right)$, i.e. infinitesimal.

The idea of constructing an "infinitesimal" permutation has been around for a long time in the replica approach to random systems. The first such transformation appeared in [23], where it was shown that a suitably chosen linear combination of replicon eigenvectors added to a Parisi vector will result in a "reparametrization" of this vector, i.e. in a modified sequence of parameters $q_{r}$ and $p_{r}$. This observation will be further developed below. Shortly thereafter Goltsev introduced a set of "infinitesimal permutations" and showed how any finite permutation can be built up from infinitesimal ones [24], without exploiting the full potential of these infinitesimal generators. Independently, Parisi and Slanina rediscovered the same transformation in a random polymer context [25]. Finally an infinitesimal transformation closely related to the one above was used by ourselves to derive Ward identities for the spin glass [19], in the rest of this section we recall some of the results derived therel.

After finding infinitesimal symmetry transformations, we can follow the usual steps to obtain Ward identities in a system with a continuous symmetry [3]. Since $\vec{q}^{\prime}$ is very close to $\vec{q}$ we can expand the left hand side of Eq. 11 to get

$$
\mathbf{O} \vec{f}-\vec{f}=\mathbf{M}\left(\vec{q}^{\prime}-\vec{q}\right)+\ldots
$$

where $\vec{f}$ is the gradient vector

$$
\vec{f}=\frac{\partial F}{\partial \vec{q}}
$$

and $\mathbf{M}$ is the mass operator with components

$$
M_{\alpha \beta, \gamma \delta}=\frac{\partial^{2} F}{\partial q_{\alpha \beta} \partial q_{\gamma \delta}}
$$

Evaluating the derivatives in Eqs. 5 and 6 at $q_{\alpha \beta}$ (a Parisi vector) we get a gradient vector $\vec{f}$ with the same Parisi-like structure, and a mass operator which has the structure of an ultrametric matrix. (Ultrametric matrices commute, by definition, with all the elements of the residual group. Their structure was studied in detail in [26].) Eq. 囵 can then be analysed by the block diagonalization procedure described in [26]. As shown in that paper, the relevant quantities representing the diagonal blocks are:

- the one-dimensional replicon (R) blocks (i.e. eigenvalues) $\lambda(r ; k, l), r=0,1, \ldots, R ; k, l=$ $r+1, r+2, \ldots, R+1$, and

- the $(R+1) \times(R+1)$-dimensional longitudinal-anomalous (LA) "kernels" of the LA blocks, $K_{k}(r, s), r, s=0,1, \ldots, R$ and $k=0,1, \ldots, R+1$.

\footnotetext{
${ }^{1}$ In Ref. [19], at editing, the equation numbering was messed up. The puzzled reader will find the correct numbering in the original cond-mat/9802166 version.
} 
The explicit expressions for $\lambda(r ; k, l)$ and $K(r, s)$ are given in [26]. After block diagonalization the various components of Eq. 4 give, to leading order in $1 / R$, the following set of equations:

$$
\begin{array}{r}
f_{r+1}-f_{r}=\left(q_{r+1}-q_{r}\right) \lambda(r+1 ; r+2, r+2)+O\left(1 / R^{2}\right) \\
\lambda(r+1 ; r+2, r+2)-\lambda(r ; r+1, r+2)=O(1 / R) \\
\lambda(r ; r+2, r+2)-\lambda(r ; r+1, r+2)=O(1 / R) \\
K_{r+2}(r, s)-K_{r+2}(r+1, s)=O(1 / R)
\end{array}
$$

with $r=0,1, \ldots, R-1$ and $s=0,1, \ldots, R$. Eq. 8 expresses a continuity property of the mass operator, while Eq. I is a Ward identity which establishes a relationship between the first and second derivatives of $F$. In the limit $R \rightarrow \infty$, after introducing the continuous parameter $x$ by

$$
x=\frac{r}{R+1} \quad, \quad 0 \leq x \leq 1
$$

(17) becomes

$$
\frac{d f(x)}{d x}=\frac{d q(x)}{d x} \lambda(x ; x, x) \quad, \quad 0<x<1 .
$$

If, finally, we take Eq. 10 at a stationary point where $f \equiv 0$, we obtain

$$
\lambda(x ; x, x)=0
$$

for all $x$ where $\frac{d q(x)}{d x} \neq 0$. (If, as is often the case, there is a breakpoint $x_{1}$ beyond which $q(x)$ and also $\lambda(x ; x, x)$ are constant, (11) still holds in the limit $x \rightarrow x_{1}^{-}$, and, by continuity, it also holds for $x>x_{1}$.) The status of Eq. 11 is that of a Goldstone theorem for spin glasses: under the assumptions of permutation invariance and the existence of the continuous limit $R \rightarrow \infty$, (11) follows, independently of the concrete form of the free energy functional $F$.

It is straightforward, at least in principle, to apply the above ideas for deriving higher order Ward identities. The mass operator $\mathbf{M}$ is the second derivative of the free energy, and thus it transforms as a tensor under an orthogonal transformation $\mathbf{O}$ :

$$
\mathbf{M}^{\prime}=\mathbf{O M O}^{\mathrm{T}}
$$

where $\mathbf{M}^{\prime}$ refers to the derivatives in (6) evaluated at $\vec{q}^{\prime}=\mathbf{O} \vec{q}$. Expanding the left hand side of Eq. 12 around $\vec{q}$, and introducing the 3 -point vertex function as

$$
W_{\alpha \beta, \gamma \delta, \mu \nu}=\frac{\partial^{3} F}{\partial q_{\alpha \beta} \partial q_{\gamma \delta} \partial q_{\mu \nu}}
$$


we get

$$
\left(\mathbf{O M O}^{\mathrm{T}}\right)_{\alpha \beta, \gamma \delta}-(\mathbf{M})_{\alpha \beta, \gamma \delta}=\sum_{\mu<\nu} W_{\alpha \beta, \gamma \delta, \mu \nu}\left(\vec{q}^{\prime}-\vec{q}\right)_{\mu \nu}+\ldots
$$

Eq. 14 is the starting point for obtaining the set of Ward-identities establishing the exact connections between components of the 2- and 3-point vertices. To accomplish this work, a procedure, similar to that of block diagonalisation of the 2-point vertices, transforming the 3 -point vertices into a "canonical" form is needed. A simple example of such a relationship between 2 and 3 -point vertices is worked out in 19].

\section{Reparametrization invariance -a subtle corollary of permu- tation symmetry}

As we see now, the symmetry responsible for the Goldstone modes is permutation invariance that becomes "continuous" in the limit $R \rightarrow \infty$. Nevertheless, in Ref. [15] two of us purported to derive (11) from a rather liberal interpretation of an other kind of symmetry: reparametrization invariance. The obviously correct result was obtained by using some uncontrollable calculational steps, such as forgetting about the differences between derivatives with respect to $x$ or $p(x)$. Having two independent symmetries leading to the same exact property of the system, i.e. the masslessness of some of the modes, is rather unlikely, and one can suspect that a connection between them must exist. In this section, we will find this connection showing how reparametrization invariance follows, in the limit $n \rightarrow 0$ and $R \rightarrow \infty$, from the primary symmetry of the equivalence of the $n$ replicas.

The first thing we need is a clear definition of the reparametrization transformation. It connects two Parisi vectors $\vec{q} \rightarrow \vec{q}^{\prime}$ with the following properties:

$$
\begin{array}{rlrl}
p_{r}^{\prime} & =p_{r}+\delta p_{r}, & & r=1, \ldots, R ; \\
q_{r}^{\prime} & =Q\left(p_{r}^{\prime}\right), & & r=1, \ldots, R ; \\
q_{0}^{\prime} & =q_{0}, &
\end{array}
$$

supplemented by the condition that the endpoints be invariant, $\delta p_{1}=\delta p_{R}=0$. The function $Q(p)$ is obtained from $q(x)$ and $p(x)$ by eliminating $x$ between them, and it remains fixed under a chain of reparametrization transformations (see Fig. 价).

At this point we wish to stress that the reparametrization invariance is not a property of the free energy functional $F\left(q_{\alpha \beta}\right)$, but of the functional $\tilde{F}\left(q_{r}, p_{r}\right)$ obtained from $F$ by restricting its argument $q_{\alpha \beta}$ to the subspace spanned by the Parisi-like vectors. This subspace may be called

longitudinal, and, by extension, $\tilde{F}$ may be called the "longitudinal" free energy. $\tilde{F}$ remains unchanged when moving from $\vec{q}$ to $\vec{q}^{\prime}$ by the reparametrization transformation of Eqs. 15.17 :

$$
\tilde{F}\left(q_{r}^{\prime}, p_{r}^{\prime}\right)=\tilde{F}\left(q_{r}+\delta q_{r}, p_{r}+\delta p_{r}\right)=\tilde{F}\left(q_{r}, p_{r}\right) .
$$




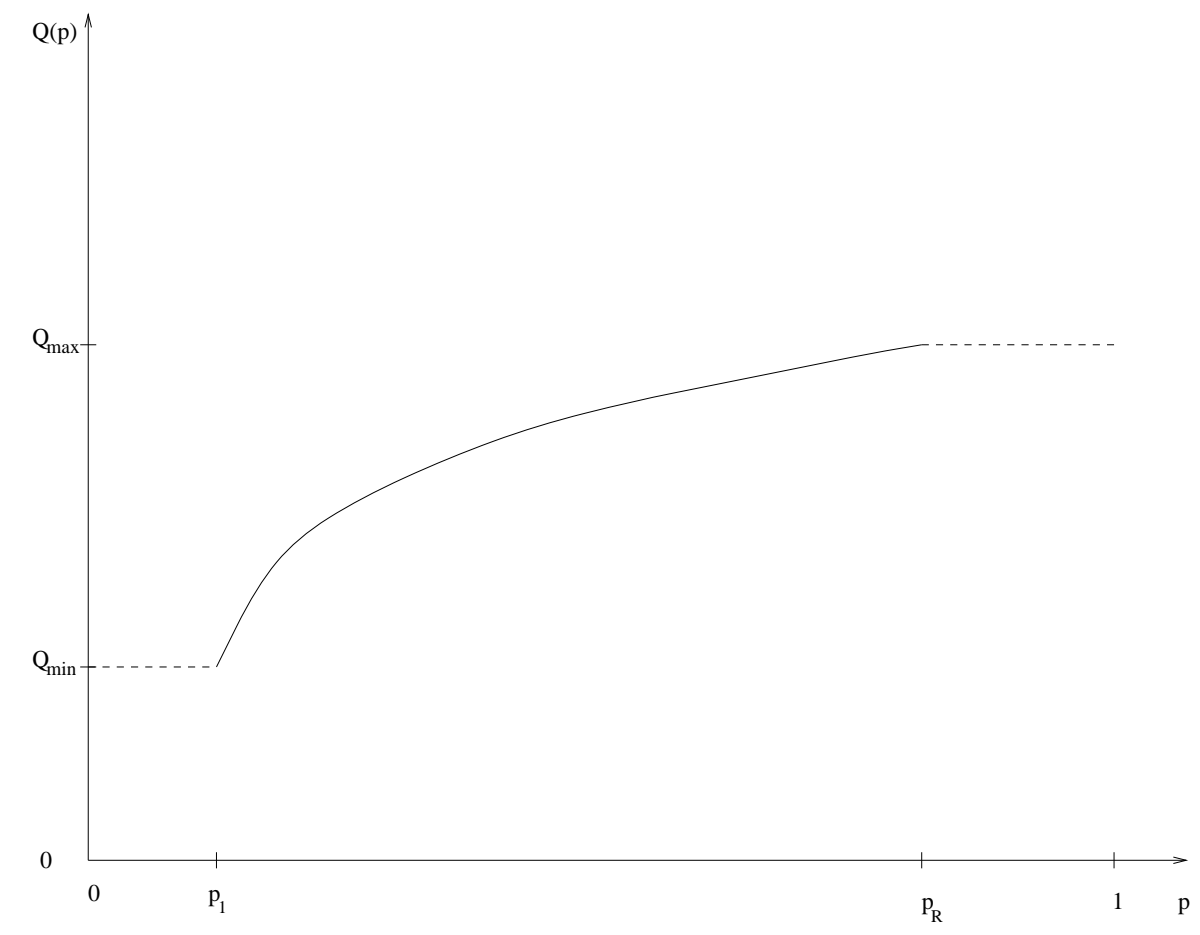

Figure 1: $Q(p)$ defines a chain of reparametrization transformations 
In the above equation $\vec{q}$ and $\vec{q}^{\prime}$ are Parisi vectors with the properties given in Eq. 2, it expresses, therefore, an exact symmetry of $\tilde{F}$ only in the limit $R \rightarrow \infty$. For finite $R$ the invariance of $\tilde{F}$ is only approximate, valid within corrections of $O(1 / R)$. Eq. 18 can be cast into an equivalent form using variations of $\tilde{F}$ :

$$
\delta^{(k)} \tilde{F}[q(x), p(x)]=0
$$

for any positive integer $k$. In Eq. 19 only $p(x)$ is varied independently, with the constraint of fixed endpoints $\delta p(0)=\delta p(1)=0$. By Eq. 16, $\delta q(x)$ can be expressed in terms of $\delta p(x)$, an expanded form of which is useful to be recorded here for further reference:

$$
\delta q(x)=Q^{\prime}[p(x)] \delta p(x)+\frac{1}{2} Q^{\prime \prime}[p(x)] \delta p(x)^{2}+\ldots \quad .
$$

To prove the invariance properties in Eqs. 18 or 19, $\Delta \tilde{F} \equiv \tilde{F}\left(q_{r}^{\prime}, p_{r}^{\prime}\right)-\tilde{F}\left(q_{r}, p_{r}\right)$ will be expanded up to second order in the $\delta q_{r}$ 's and $\delta p_{r}$ 's. A simple Taylor expansion yields

$$
\Delta \tilde{F}=\sum_{r=1}^{R}\left(\frac{\partial \tilde{F}}{\partial q_{r}} \delta q_{r}+\frac{\partial \tilde{F}}{\partial p_{r}} \delta p_{r}\right)+\frac{1}{2} \sum_{r, s=1}^{R}\left(\frac{\partial^{2} \tilde{F}}{\partial q_{r} \partial q_{s}} \delta q_{r} \delta q_{s}+2 \frac{\partial^{2} \tilde{F}}{\partial q_{r} \partial p_{s}} \delta q_{r} \delta p_{s}+\frac{\partial^{2} \tilde{F}}{\partial p_{r} \partial p_{s}} \delta p_{r} \delta p_{s}\right) .
$$

On the other hand, the displacement between the two "longitudinal" (Parisi-type) vectors in the full vector space of the $q_{\alpha \beta}$ 's gives an equivalent form for $\Delta \tilde{F}$. Using the short-hand notation of brackets as in the Appendix (Eqs. 33, 34), we can write

$$
\Delta \tilde{F}=\left\langle f \mid q^{\prime}-q\right\rangle+\frac{1}{2}\left\langle q^{\prime}-q|M| q^{\prime}-q\right\rangle+\ldots
$$

$\vec{f}$ and $\mathbf{M}$ were defined in Eqs. 5 and 6, and under any permutation $P$ of the replicas, corresponding to an orthogonal transformation $\mathbf{O}$ in the order parameter space of the $q_{\alpha, \beta}$ 's, they transform according to Eqs. 11 and 12, respectively. As pointed out in Sec. 2, this is a clear consequence of the permutation invariance of the free energy $F\left(q_{\alpha \beta}\right)$. Furthermore, taking the permutation $P$ from the residual symmetry group corresponding to the ultrametric construction of the vector $\vec{q}$ (which is obviously different from the group of transformations defined by $\vec{q}^{\prime}$ ), i.e. $\mathbf{O} \vec{q}=\vec{q}$, we get

$$
\vec{f}^{\prime}=\vec{f}=\mathbf{O} \vec{f} \quad \text { and } \quad \mathbf{M}^{\prime} \mathbf{O}=\mathbf{M O}=\mathbf{O M} .
$$

Thus $\vec{f}$ and $\mathbf{M}$ are ultrametric, and Eqs. 36,39 of the Appendix can be used in Eq. 22. Putting everything together from the Appendix (especially from Eqs. 31, 32, 38 and 40), a comparison of Eqs. 21 and 22 yields:

$$
\begin{array}{ll}
\frac{\partial \tilde{F}}{\partial q_{r}}=\frac{n}{2}\left(p_{r}-p_{r+1}\right) f_{r} & r=0, \ldots, R \\
\frac{\partial \tilde{F}}{\partial p_{r}}=\frac{n}{2}\left(q_{r}-q_{r-1}\right)\left[f_{r}-\frac{1}{2}\left(q_{r}-q_{r-1}\right) \lambda(r ; r+1, r+1)\right] & r=1, \ldots, R
\end{array} .
$$


From this, in the limit $R \rightarrow \infty$, the first variation $\delta^{(1)} \tilde{F}$ follows immediately. Introducing the continuous variable $x$ (see Eq. 9):

$$
\delta^{(1)} \tilde{F}=\frac{n}{2} \int_{0}^{1} d x\left[q^{\prime}(x) f(x)-Q^{\prime}[p(x)] p^{\prime}(x) f(x)\right] \delta p(x) \quad ;
$$

in deriving the above expression, use has been made of Eqs. 21 and 24, together with the reparametrization constraint Eq. 20.

The integrand in Eq. 25 is identically zero, which is a direct consequence of the definition of $Q(p): Q[p(x)]=q(x)$. Thus the disappearence of the first variation, $\delta^{(1)} \tilde{F}=0$, seems to be somewhat trivial. We must emphasize, however, that it is true along the whole chain of reparametrization transformations; a result which follows from the crucial step in the proof: if $F\left(q_{\alpha \beta}\right)$ is invariant for any permutations of the replicas, then its derivatives taken at an ultrametrically structured $\vec{q}$ are themselves ultrametric. The permutation group of the $n$ replicas is "large" enough to include all the subgroups defined by the Parisi-type vectors along a path of consecutive infinitesimal reparametrization transformations. $\delta^{(1)} \tilde{F}$ is, therefore, identically equal to zero, implying the two equivalent form of reparametrization invariance, Eqs. 18 and 19 .

With its proof now accomplished, we can proceed and use Eq. 19 for $k=2,3, \ldots$ to derive Ward-identities (in principle to any desired order). They must not be different from those following from the primary symmetry of permutation invariance using infinitesimal permutations, as in Sec. 2. To show this, we compute $\delta^{(2)} \tilde{F}$. The second partial derivatives of $\tilde{F}$ can be calculated, by intensive use of the Appendix, just like the first ones were in Eq. 24. The results are as follows:

$$
\begin{aligned}
\frac{\partial^{2} \tilde{F}}{\partial q_{r} \partial q_{s}}= & \frac{n}{4}\left(p_{r}-p_{r+1}\right)\left(p_{s}-p_{s+1}\right) K_{0}(r, s)+\frac{n}{2}\left(p_{r}-p_{r+1}\right) \lambda(r ; r+1, r+1) \delta_{r, s}^{\mathrm{Kr}} \\
\frac{\partial^{2} \tilde{F}}{\partial p_{r} \partial q_{s}}= & \frac{n}{4}\left(q_{r}-q_{r-1}\right)\left(p_{s}-p_{s+1}\right) K_{0}(r, s)+\frac{n}{2} f_{r} \delta_{r, s}^{\mathrm{Kr}}+ \\
& +\frac{n}{2}\left[-f_{r}+\left(q_{r}-q_{r-1}\right) \lambda(r ; r+1, r+1)\right] \delta_{r-1, s}^{\mathrm{Kr}} \\
& r=1, \ldots, R \quad \text { and } \quad s=0, \ldots, R \quad, \\
\frac{\partial^{2} \tilde{F}}{\partial p_{r} \partial p_{s}}= & \frac{n}{4}\left(q_{r}-q_{r-1}\right)\left(q_{s}-q_{s-1}\right) K_{0}(r, s) \\
& r, s=1, \ldots, R .
\end{aligned}
$$

It is now straightforward to compute $\delta^{(2)} \tilde{F}$ using Eqs. 20,21,24 and 26. Note that the second term in Eq. 20 multiplied by the first partial derivative of $F$ with respect to $q(x)$ also contributes. The rather lengthy calculation consists of mainly partial integrations, surface terms always 
disappearing because of the constraint $\delta p(0)=\delta p(1)=0$. After a lot of simplification, the $K_{0}(x, y)$ terms cancel each other, yielding the surprisingly simple result:

$$
\delta^{(2)} \tilde{F}=\frac{n}{2} \int_{0}^{1} d x\left[q^{\prime}(x) \lambda(x ; x, x)-f^{\prime}(x)\right] Q^{\prime}[p(x)] \delta p(x)^{2}
$$

Since $\delta p(x)$ is arbitrary, and assuming a monotonic $Q(p)$, the vanishing of the second variation is equivalent with the vanishing of the expression in the brackets in Eq. 27; i.e. Eq. 10 is now regained from the invariance of the free energy $\tilde{F}$ when the "gauge" $p(x)$ is changing.

An example of two equivalent "gauges" with fixed endpoints is shown in Fig. 2. We can even deform curve (b) to curve (c) in Fig. 3 with the plateaux around $x=0$ and $x=1$. Supplementing the definition of $Q(p)$ by the plateaux regions drawn by dashed lines in Fig. 1, we can easily figure out that the parametrization of curve (d) represents the same Parisi vector $q_{\alpha \beta}$ as curve (c). In that case, $q(x)$ has also two plateaux with $Q_{\min }$ and $Q_{\max }$. Thus, the "longitudinal" free energy remains invariant for reparametrizations where the endpoints $p(0)$ and $p(1)$ move apart: $0 \leq p(0) \leq p_{1}$ and $p_{R} \leq p(1) \leq 1$. The most common gauge, $p(x)=x$, introduced by Parisi [B], is also displayed as curve (e) in Fig. 3 .

As a last remark, we want to stress that reparametrization invariance expresses simply the fact that it is not the gauge $p(x)$ but $Q(p)$ which has a physical meaning. (For the Ising spin glass, the probability distribution of pures states is the derivative of the inverse function $p(Q)$.)

\section{Acknowledgment}

This work has been supported by the Hungarian Science Fund (OTKA), Grant No. T032424, and by the convention CEA/MAE 2000.

\section{Appendix}

In the starting phase of Parisi's ultrametric construction (see the references [8, 16]) the block sizes $p_{r}$ 's are large integers with the property that each $p_{r+1}$ is a divisor of $p_{r}$ for all $r=0,1, \ldots, R$ and the continuum of the $p_{r}$ 's with the inverted monotonicity of a Parisi vector, defined in Sec. 2, is obtained after taking $n \rightarrow 0, R \rightarrow \infty$. (For a more mathematical treatment, see [27].) We adopt a similar construction in the reparametrization procedure and consider $p_{r+1}$ a common divisor of $p_{r}^{\prime}$ and $p_{r}$, while $p_{r}^{\prime}$ itself is a divisor of $p_{r}$; of course, this is valid at the stage before the "upside down" continuation of $n$ from a large integer to zero, and $\delta p$ of Eq. 15 can be considered an arbitrary function of $x$ with the only condition $\delta p(0)=\delta p(1)=0$.

In this appendix, however, we remain in the domain of large integer block sizes $p_{r}$ and $p_{r}^{\prime}$, for all $r=0,1, \ldots, R$ and finite $R$, and the properties of the previous paragraph will be assumed. This enables us to expand the vector $\vec{q}^{\prime}-\vec{q}$, the displacement under the reparametrization 


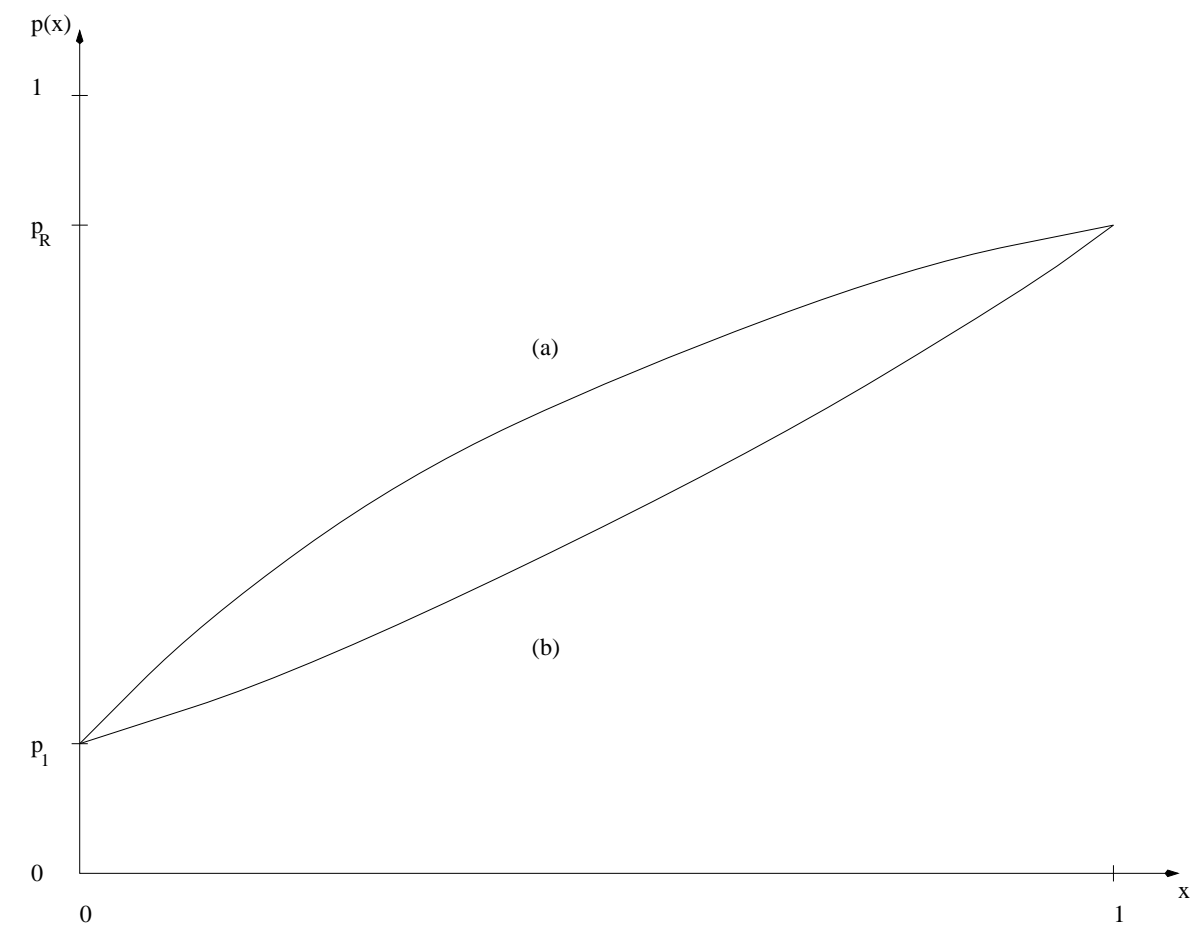

Figure 2: Reparametrization with fixed endpoints 


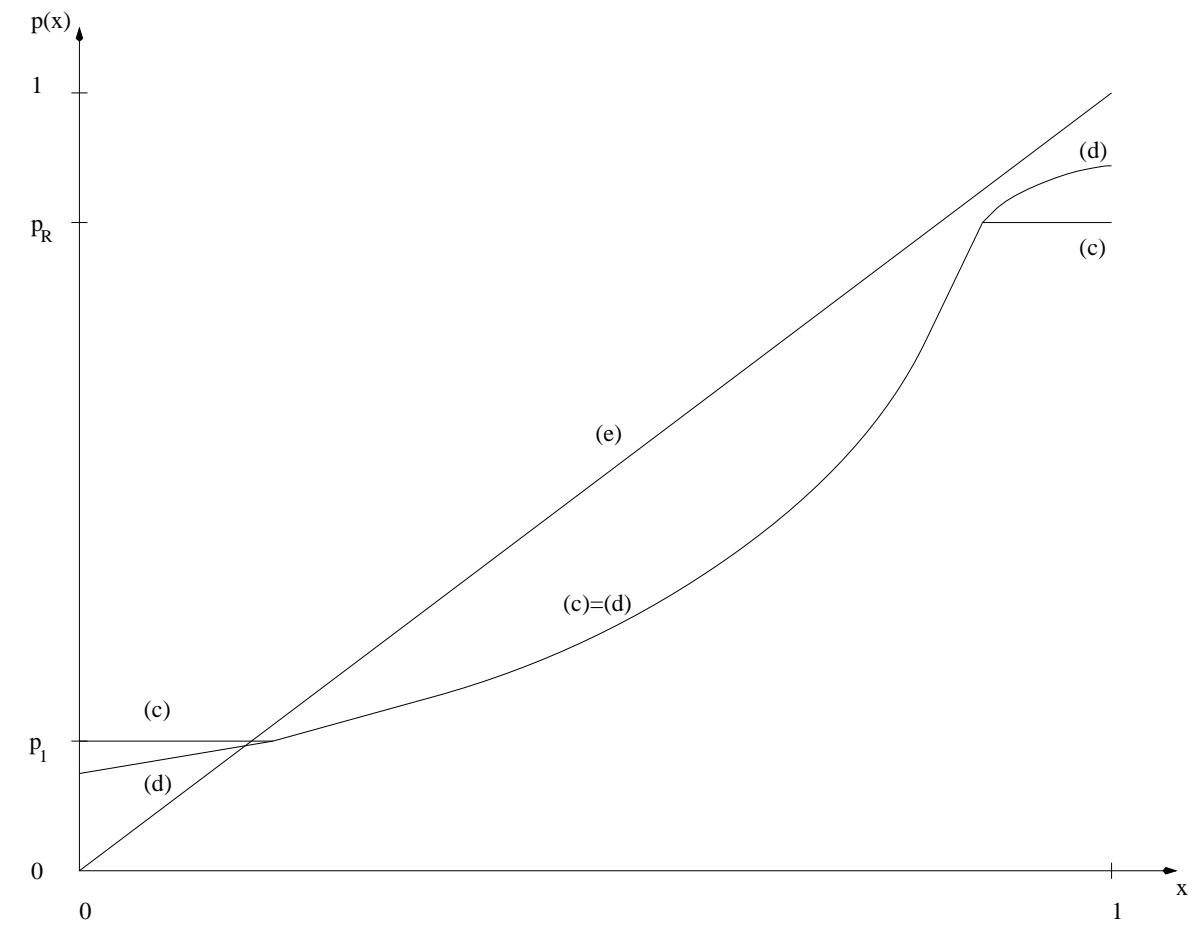

Figure 3: Extending reparametrization transformation to gauges with moving endpoints (see text) 
transformation, in terms of basis vectors. These basis vectors form a complete set in the $\frac{n(n-1)}{2}$ dimensional space of the $q_{\alpha \beta}$ 's, and they block-diagonalize any generic ultrametric matrix. (For a detailed analysis of the structure of this non-orthogonal basis, see [26].) Following [23], we can figure out that only two types of basis vectors are involved in the expansion: a replicon one $\vec{q}^{(\mathrm{R}, r)}$ and a longitudinal one $\vec{q}^{(\mathrm{L}, r)} \cdot \vec{q}^{(\mathrm{R}, r)}$ is just the linear combination of the $(r ; r+1, r+1)$ modes which shows up in Eq. (7) of [23], and as such, it is an eigenvector of any ultrametric matrix with the eigenvalue $\lambda(r ; r+1, r+1)$. It has nonzero components only for $\alpha \cap \beta=r$, taking two different values depending on the overlap of $\alpha$ and $\beta$ with respect to the ultrametric structure defined by the new block sizes $p_{r}^{\prime}$-s:

$$
\begin{aligned}
& \vec{q}_{\alpha \beta}^{(\mathrm{R}, r)}=\frac{\left(p_{r}-2 p_{r+1}\right)\left(p_{r}-p_{r}^{\prime}\right)}{2 p_{r+1}^{2}}, \quad \alpha, \beta \text { in the same } p_{r}^{\prime} \text { block } \\
& \vec{q}_{\alpha \beta}^{(\mathrm{R}, r)}=-\frac{\left(p_{r}-2 p_{r+1}\right)\left(p_{r}^{\prime}-p_{r+1}\right)}{2 p_{r+1}^{2}}, \quad \alpha, \beta \text { in different } p_{r}^{\prime} \text { blocks. }
\end{aligned}
$$

As for the longitudinal vector $\vec{q}^{(\mathrm{L}, r)}$, it has zero elements everywhere, except for $\alpha \cap \beta=r$ :

$$
\begin{array}{ll}
\vec{q}_{\alpha \beta}^{(\mathrm{L}, r)}=1, & \alpha \cap \beta=r ; \\
\vec{q}_{\alpha \beta}^{(\mathrm{L}, r)}=0, & \alpha \cap \beta \neq r .
\end{array}
$$

$r=0,1, \ldots, R$ in Eq. 29, while, remembering that $p_{0}^{\prime}=p_{0}=n$, the replicon vector of Eq. 28 is not defined for $r=0$.

The displacement vector $\vec{q}^{\prime}-\vec{q}$ can be expanded as

$$
\vec{q}^{\prime}-\vec{q}=\sum_{r=1}^{R}\left(K^{(\mathrm{R}, r)} \vec{q}^{(\mathrm{R}, r)}+K^{(\mathrm{L}, r)} \vec{q}^{(\mathrm{L}, r)}\right)+\left(q_{0}^{\prime}-q_{0}\right) \vec{q}^{(\mathrm{L}, 0)}
$$

with the coefficients $K^{(\mathrm{R}, r)}$ and $K^{(\mathrm{L}, r)}$ determined from the conditions

$$
\begin{array}{rlrl}
\left(\vec{q}^{\prime}-\vec{q}\right)_{\alpha \beta} & =q_{r}^{\prime}-q_{r} & & \alpha, \beta \text { in the same } p_{r}^{\prime} \text { block } \\
\left(\vec{q}^{\prime}-\vec{q}\right)_{\alpha \beta} & =q_{r-1}^{\prime}-q_{r} & \alpha, \beta \text { in different } p_{r}^{\prime} \text { blocks }
\end{array}
$$

for $r=\alpha \cap \beta=1, \ldots, R$. (The $r=0$ case is trivial, with $\left(\vec{q}^{\prime}-\vec{q}\right)_{\alpha \beta}=q_{0}^{\prime}-q_{0}$, leading to the simple last term in Eq. 30.) Using Eqs. 28,29, it is straightforward to obtain $K^{(\mathrm{R}, r)}$ and $K^{(\mathrm{L}, r)}$. For later reference, it is useful to express them in terms of $\delta q_{r}=q_{r}^{\prime}-q_{r}$ and $\delta p_{r}=p_{r}^{\prime}-p_{r}$ :

$$
\begin{aligned}
K^{(\mathrm{R}, r)} & =\frac{2 p_{r+1}^{2}}{\left(p_{r}-2 p_{r+1}\right)\left(p_{r}-p_{r+1}\right)}\left[\left(q_{r}-q_{r-1}\right)+\delta q_{r}-\delta q_{r-1}\right], \\
K^{(\mathrm{L}, r)} & =\delta q_{r}-\frac{q_{r-1}-q_{r}}{p_{r}-p_{r+1}} \delta p_{r}-\frac{1}{p_{r}-p_{r+1}}\left(\delta q_{r-1} \delta p_{r}-\delta q_{r} \delta p_{r}\right) .
\end{aligned}
$$


What we need in the main text, is the scalar product

$$
\left\langle f \mid q^{\prime}-q\right\rangle \equiv \sum_{\alpha<\beta} f_{\alpha \beta}\left(q^{\prime}-q\right)_{\alpha \beta}
$$

and the matrix elements

$$
\left\langle q^{\prime}-q|M| q^{\prime}-q\right\rangle \equiv \sum_{\alpha<\beta, \gamma<\delta}\left(q^{\prime}-q\right)_{\alpha \beta} M_{\alpha \beta, \gamma \delta}\left(q^{\prime}-q\right)_{\gamma \delta}
$$

for an ultrametrically structured vector $\vec{f}$ and matrix $\mathbf{M}$. Since $\vec{f}$ is now a longitudinal vector (orthogonal to any replicon one), and also using Eq. 29, it follows:

$$
\begin{aligned}
\left\langle f \mid q^{(\mathrm{R}, r)}\right\rangle & =0, \\
\left\langle f \mid q^{(\mathrm{L}, r)}\right\rangle & =\frac{n}{2}\left(p_{r}-p_{r+1}\right) f_{r} .
\end{aligned}
$$

Combining Eqs. 30 and 35,

$$
\left\langle f \mid q^{\prime}-q\right\rangle=\frac{n}{2} \sum_{r=1}^{R}\left(p_{r}-p_{r+1}\right) f_{r} K^{(\mathrm{L}, r)}+\frac{n}{2}\left(n-p_{1}\right) f_{0} \delta q_{0}
$$

obtains. To find a similar formula for the matrix element, we must use results from Ref. [26]:

$$
\begin{aligned}
& \mathbf{M} \vec{q}^{(\mathrm{R}, r)}=\lambda(r ; r+1, r+1) \vec{q}^{(\mathrm{R}, r)}, \\
& \mathbf{M} \vec{q}^{(\mathrm{L}, r)}=\sum_{s=0}^{R} M_{s, r}^{(0)} \vec{q}^{(\mathrm{L}, s)},
\end{aligned}
$$

where the block matrix element $M_{s, r}^{(0)}$ can be expressed by the longitudinal kernel $K_{0}(s, r)$ using Eq. 44 of Ref. [26]:

$$
M_{s, r}^{(0)}=\lambda(r ; r+1, r+1) \delta_{s, r}^{\mathrm{Kr}}+\frac{1}{2}\left(p_{r}-p_{r+1}\right) K_{0}(s, r) .
$$

From Eqs. 30 and 37, it is now straightforward to find the following expression for the matrix elements:

$$
\begin{aligned}
& \left\langle q^{\prime}-q|M| q^{\prime}-q\right\rangle= \\
& \sum_{r=1}^{R} K^{(\mathrm{R}, r)^{2}} \lambda(r ; r+1, r+1)\left\langle q^{(\mathrm{R}, r)} \mid q^{(\mathrm{R}, r)}\right\rangle+\sum_{r, r^{\prime}=1}^{R} K^{(\mathrm{L}, r)} K^{\left(\mathrm{L}, r^{\prime}\right)} M_{r, r^{\prime}}^{(0)}\left\langle q^{(\mathrm{L}, r)} \mid q^{(\mathrm{L}, r)}\right\rangle+ \\
& +\delta q_{0} \sum_{r=1}^{R} K^{(\mathrm{L}, r)}\left(M_{r, 0}^{(0)}\left\langle q^{(\mathrm{L}, r)} \mid q^{(\mathrm{L}, r)}\right\rangle+M_{0, r}^{(0)}\left\langle q^{(\mathrm{L}, 0)} \mid q^{(\mathrm{L}, 0)}\right\rangle\right)+\delta q_{0}^{2} M_{0,0}^{(0)}\left\langle q^{(\mathrm{L}, 0)} \mid q^{(\mathrm{L}, 0)}\right\rangle .
\end{aligned}
$$


The scalar products occuring in the above formula can be easily computed from the definitions in Eqs. 28 and 29:

$$
\begin{aligned}
\left\langle q^{(\mathrm{R}, r)} \mid q^{(\mathrm{R}, r)}\right\rangle & =-\frac{n\left(p_{r}-2 p_{r+1}\right)^{2}\left(p_{r}-p_{r+1}\right)}{8 p_{r+1}^{3}}\left(\frac{p_{r}-p_{r+1}}{p_{r+1}} \delta p_{r}+\frac{1}{p_{r+1}} \delta p_{r}^{2}\right) \\
\left\langle q^{(\mathrm{L}, r)} \mid q^{(\mathrm{L}, r)}\right\rangle & =\frac{n\left(p_{r}-p_{r+1}\right)}{2} .
\end{aligned}
$$

\section{References}

[1] J. Goldstone, Nuovo Cim. 19, 154 (1961).

[2] J. Goldstone, A. Salam and S. Weinberg, Phys. Rev. 127, 965 (1962).

[3] See e.g. J. Zinn-Justin, Quantum Field Theory and Critical Phenomena, Clarendon Press, Oxford (1989).

[4] E. Brézin, D.J. Wallace and K.G. Wilson, Phys. Rev. B 7, 232 (1973).

[5] D.J. Thouless, P.W. Anderson and R.G. Palmer, Philos. Mag. 35, 593 (1977).

[6] A.J. Bray and M.A. Moore, J. Phys. C 12, L441 (1979).

[7] D. Sherrington and S. Kirkpatrick, Phys. Rev. Lett. 35, 1792 (1975).

[8] G. Parisi, Phys. Rev. Lett. 43, 1754 (1979); J. Phys. A 13, L115 (1980); 13, 1101 (1980); 13, 1887 (1980).

[9] H. Sompolinsky, Phys. Rev. Lett. 47, 935 (1981).

[10] A.V. Goltsev, J. Phys. A 17, 237 (1984).

[11] C. De Dominicis and I. Kondor, Phys. Rev. B 27, 606 (1983).

[12] W.L. McMillan, J. Phys. C 17, 3179 (1984).

[13] D.S. Fisher and D. Huse, Phys. Rev. Lett. 56, 1601 (1986); Phys. Rev. B 38, 386 (1988).

[14] A.J. Bray and M.A. Moore, in Proceedings of the Heidelberg Colloquium on Glassy Dynamics (Lecture Notes in Physics 275), eds. J.L. van Hemmen and I. Morgenstern (Springer, Heidelberg, 1986); Phys. Rev. Lett. 58, 57 (1987).

[15] I. Kondor and C. De Dominicis, Europhys. Lett. 2, 617 (1986). 
[16] For a review see: M. Mézard, G. Parisi and M.A. Virasoro, Spin Glass Theory and Beyond (World Scientific, Singapore, 1987); K. Binder and A.P. Young, Rev. Mod. Phys. 58, 801 (1986).

[17] D.M. Carlucci, C. De Dominicis and T. Temesvári, J. Phys. I (France) 6, 1031 (1996).

[18] A.J. Bray and M.A. Moore, J. Phys. C 12, 79 (1979).

[19] C. De Dominicis, T. Temesvári and I. Kondor, J. Phys. IV (France) 8, 6 (1998).

[20] J. Luttinger and J. Ward, Phys. Rev. 118, 1417 (1960); C. De Dominicis, J. Math. Phys. 4, 255 (1963).

[21] M. Mézard and A.P. Young, Europhys. Lett. 18, 653 (1992); C. De Dominicis, H. Orland and T. Temesvári, J. Phys. I (France) 5, 987 (1995).

[22] M. Mézard and G. Parisi, J. Phys. I (France) 1, 809 (1991); 2, 2231 (1992).

[23] I. Kondor and R. Németh, Acta Phys. Hung. 62, 219 (1987).

[24] A.V. Goltsev, J. Phys. A 24, 307 (1991).

[25] G. Parisi and F. Slanina, Eur. Phys. J. B 8, 603 (1999).

[26] T. Temesvári, C. De Dominicis and I. Kondor, J. Phys. A 27, 7569 (1994).

[27] G. Parisi and N. Sourlas, Eur. Phys. J. B 14, 535 (2000). 